\title{
List of Parameters Influencing the Pedestrian Movement and Pedestrian Database
}

\author{
Mohamed H. Dridi ${ }^{1}$ \\ ${ }^{1}$ Unversity Stuttgart, Institute für Theoretische Physik, Germany. \\ Correspondence: Mohamed H. Dridi, Unversity Stuttgart, Institute für Theoretische Physik, Deutschland.
}

Received: May 13, 2015

Accepted: May 27, 2015

Available online: June 8, 2015

doi:10.11114/ijsss.v3i4.870

URL: http://dx.doi.org/10.11114/ijsss.v3i4.870

\begin{abstract}
In this paper we present a list of factors influencing the pedestrian behavior in different situations and conditions. In crowd simulation input we must consider at least two simulation conditions. The first is the normal condition and the second is the emergency condition or panic situation. In panic situations most parameters will be changed and the time factor becomes very important. Both emotion and personality clearly have a strong and considerable impact on individual behaviors in such situations. However, most existing approaches in their attempt to model the behavior of individuals and for guiding an agent to interact with its environment and other agents, consider the individual as an autonomous agent or autonomous particle that obeys some human-like behavior modules such as locomotion, perception, and decision making. Other models treat the crowd as a collection of homogeneous particles interacting through physical forces. Today with the enormous knowledge development in computer science, many models try to improve themselves. There seems to be an evolution in crowd simulation to model each individual as some kind of intelligent agent with attempts to incorporate more and more social and psychological factors into the agent behavior model. However, to reproduce more realistic simulation behaviors many factors and attributes influencing pedestrians must be considered. The actual shortcoming of the existing models is the absence of modeling the social group process and its impact on human behavior. One way to gain a better understanding of human behavior in this area is to enrich the tools available for planning, such as pedestrian micro simulation in case of panic situations and emergency conditions. In this work a pedestrian database called PedGUI containing a lot of information about pedestrians is developed, this have a significant impact on the simulation input at least two mean pedestrian characteristics like age and gender can be considered.
\end{abstract}

Keywords: Pedestrian Dynamics - Crowd Simulation and Modeling, Crowd management and pedestrian safety, Crowd psychology, Pedestrian parameters, Ethnic groups and culture parameters, Pedestrian database, Hajj simulation.

\section{Introduction}

Today's crowd simulation technology is not well adapted or compatible to the real world. Unfortunately, very little is known about the characteristics and behavior of pedestrians in cases of high density crowd dynamics. There are various parameters and factors affecting the behavior of individuals, these parameters must be considered in modeling the rules of interaction between the individual and their environment, as well as between the individual and other individuals.

In order to achieve more realistic crowd behaviors, scientists must pay attention to a lot of parameters and factors affecting the movement of the pedestrians when creating their models (e.g. weather, age of the subjects, physical fitness,... ). The movement of a single individual at any time is a result of a long list of possible (and very likely conflicting) forces and circumstances. Among these, we mention the motivation to reach a certain place at a certain time. This depends on the will force of the person and the surrounding conditions in the field of movement. The will force can be affected by many parameters, such as the fitness condition of the pedestrian. The parameters influencing the desired velocity and the relaxation time of individuals (e.g. the environmental factors and the age of the person) have a significant effect on the time needed for the individual to reach a certain place (a young pedestrian reaches his point of interest faster than an older pedestrian). Time constraints, the importance of punctuality, location constraints and the importance of reaching a certain place are the results of an individual's inner and outer factors.

In the real world the pedestrian walking speeds are influenced by many physical and psychological factors e.g. environmental surrounding, traffic flow, and pedestrian characteristics. Walking speeds can be affected by the terrain 
condition, although it can be expected that old walkers would be influenced more when walking up or down stairs than the young walkers. Similarly, it can be expected that the older persons would be more careful in case of higher crowd densities and traffic speeds out of a fear of traffic. Finally, pedestrian speed on pavements and cross ways are strongly related to the number of pedestrians in the flow. The relationship between speed, flow, and space occupied (i.e. density) for a representative population group has been examined by Predtetschenski [1], Fruin [2], Seyfried [3], and others.

The new simulation model will consider individual behaviour and independent characteristics (age, sex, size, health, body language, ethnic origin, psychic state of the individual, psycho social interaction) while also taking into account crowd-level features (e.g. panic), and characteristics of the environment (e.g. safety levels). The simulations performed will model motion and emotion.

\section{Types of Crowds}

In real life, concerning the behaviour of human beings at any time, we can distinguish different types of crowds; every type of crowd has a specific property that must be considered in the simulation model.

a)Ambulatory crowd - People walk in and out or to and from a point of interest to accomplish their daily duties e.g. working and shopping. (Example: normal streets and public places, traffic stations).

b)Crowd of spectators - People attending a game/event, we distinguish two type of spectators, first type not to communicate with each other (example: Cinema, Theater). Other type ofspectators interact with other (example: soccer game or concert);

c)Participatory crowds - People participate in the task (example: ritual activities).

d)Expressive or revelling crowds - People let their emotions out (example: demonstrations).

e)Panic crowd - Evacuations from air planes, ships and trains.

f) Special case crowd - Huge people gathering in the same place at the same time (Hajj: places of pilgrimage and worship with up to 3.5 Million pilgrims in Mecca and the Love Parade as very famous example with up to 1.5 million people).

These crowd classifications were published by the International Foundation for Protection Officers[4].

\subsection{Panic Condition (Emergency)}

Panic situations are one of the most studied and investigated phenomena in collective behaviors $[5,6,7,8]$, as they often lead to panic attacks and hence are responsible for the deaths of many people. This effect is caused by natural catastrophes (earthquakes, fires,...) or by humans themselves (emotional instability, fear, spatial limitations, demographic factors,...). Fire can be very dangerous or deadly and if the evacuation plan in the workplaces or generally in closed areas are not easy to follow, panic is inevitable. People are emotional, their emotion depends on factors, such as culture and weather. Another important panic parameter is fear. The fear of hazard or damage can evolve from emotions related to panic and may have deadly consequences. Act of violence propagate in a crowd when people feel anger or highly stressed. Spatial limitations can have dramatic consequences in panic situations. In an attempt to escape a dangerous area, there must be an available amount of space for every pedestrian to exit the facility safely. Many studies show that the demographic problems can be dangerous for crowd populations. Specific locations or groups of people cause crisis events. Depending on the nature of the event, it can create an environment for possible aggressive behavior, such as demonstrations (e.g. anti-war or anti-abortion) that the emotional surrounding of the protesters can developing disorder and violent situations. Another example which occurs frequently are fights between fans of rivaling soccer clubs after a match. Unfortunately, the number of such disasters increased worldwide over recent years (Love Parade, Pilgrimage places, soccer arenas,....). The growing population density, and therefore increased huge evolution of transport systems, leads to bigger mass events like pop concerts, sport events, and demonstrations.

Some characteristics of panic, according to Helbing et al. [9] are described in the following paragraph. In dangerous situations, individuals are excited and everybody gets nervous, i. e. they tend to develop inconvenient actions. People try to move considerably faster than normal [1]. Individuals start pushing and interactions between people become physical in nature. However, movements through bottlenecks frequently become uncoordinated, often this leads to blockade of the passage [10]. Jams occur at exits [10]. Sometimes, clogging effects are observed in intermittent flows [1], see figure 1. Crowd force can be fatal and can reach a high dangerous level, it rises with accumulate number of pedestrians in jammed crowds and can cause dangerous pressures of up to 4,500 Newtons per meter [11,12], which can built steel barriers that nobody can escape. The magnitude and direction of interaction forces between members of a large crowd can suddenly change [13], caused throwing people around in all directions which may cause trampling of

people. Trampling or falling people on the ground become a gravely "obstacle" and slow down the movement of the rest of the pedestrians. Usually in such situations people tend to show herding behaviour, i.e. to do what other people do [14, 
7]. Shocked by the panic behaviour individuals can easily overlook alternative exits or forget the escape plan [14, 12].

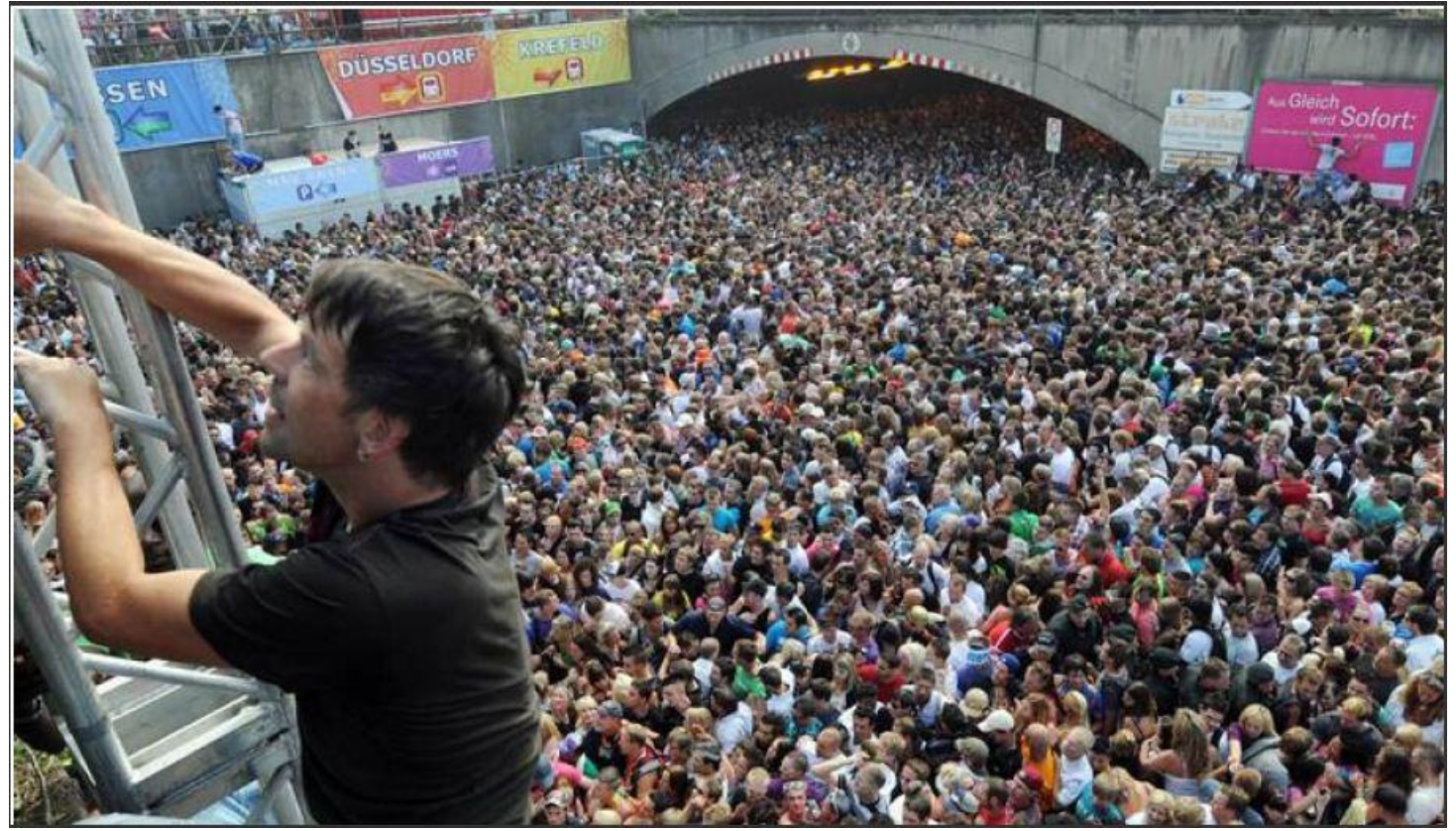

Figure 1: High density crowd on the Love Parade in Duisburg Germany. People try to escape the dangerous area to protect themselves. The local police in Duisburg reported on Saturday, July 24 2010, that at least 19 people was killed when crowd mass panic and collapse breaks out. In the area before a tunnel leading to the event plain many people were crushed and trampling to death, (EPA).

\section{List of Parameters Governing the Pedestrian Movement}

\subsection{Ethnic Groups and Culture Parameters}

Ethnic descent and cultural origins intensively affect human behaviour, especially in the case of collective games such as soccer or street demonstrations. Different cultures mean different social norms what can be identified as normal behaviour in one culture may be regarded undesirable by another [15]. Although since the old civilizations the world has a wide diversity of cultural traditions, that are accomplishing or fighting against each other, there are some kinds of behavior (such as incest, violence against clans, theft and rape) that are considered inadmissible in almost all of them. There are, however, considerable analogies in how individuals will be influenced by the same pattern or lifestyle i.e. growing up in the same culture. Furthermore, culturally induced behavior patterns can influence the movement of people in celebration places where people from different countries and cultural origins meet (like the Hajj event in Mecca). The personality and behaviour of each individual is a results of genetic inheritance and the accumulate experience in the society. Moreover the characteristics of each person is affected by a list of social and cultural circumstances (e.g. language, religion and school). Although the most social connections of persons can be traced back to their social rank [15].

These facts about the social behaviour of individuals in their social environment motivated scientists to perform experiments on the behaviour of people of different cultural origins in crowded places. Chattaraj et. al. [16] studied walking speeds of pedestrians with different cultural backgrounds in a corridor. The walking speed of Indian test persons is less related to the density then for German test persons. Surprisingly the disorganized behavior of Indians is more effective than the ordered behaviour of Germans. By statistical measurements and quantitative comparison it is confirmed that these differences exist and can be traced back to the cultural differences in the fundamental diagram of the pedestrians [16]. Till this moment the relation between pedestrian walking speed and density are not clearly understood neither the parameters influencing the pedestrian dynamics. Even in case of a simple pedestrian flow (walking a corridor) this fundamental relation is not completely agreed. For this sake we were working to better understand the characteristics of the pedestrian movement and how, for example cultural attributes influence the fundamental diagrams.

We consider the above-mentioned results as very important to simulate, as people with different ethnics and cultural origin are found in crowds everywhere like in Hajj in Saudi Arabia or at soccer matches. The pilgrims arrive from more than 100 different countries (Africa, Americas, Asia and Europe). Hundreds of thousands of them are illiterate, and they 
speak dozens of different languages. In some of their countries, the custom is to walk on the right side of the street; in others, the custom is to walk on the left. The situation involves "many uncontrollable variables" which were noted by Dirk Helbing [13].

For this reason we mention that the ethic cultural parameter is very important to be considered in simulating more realistic crowd behaviour.

\subsection{Age Parameter and Subject Selection}

Many investigations and studies on the walking speeds of young and old pedestrians, at different locations and under several different environmental conditions, indicate that the mean walking speed is affected by the age parameter. Richard L. Knoblauch in his field studies of pedestrian walking speed and start-up time [17], shows that location and environmental factors collected indicate a significant effect caused by age. Many observations carried in different sites or location showed a significant interaction between pedestrian age and the location factor. The causes of slow movement by older people have been attributed to muscle weakness among other factors.

Our database contained different data concerning the pedestrian walking speed, taking sex and age into account and whether the pedestrians were walking alone or in groups. Data was collected respecting different kinds of pedestrian ages and sex. The following individuals were specifically interesting for our investigation on pilgrimage places:

a)Pedestrians carrying children, heavy bags, or suitcases;

b)Pedestrians pushing wheelchairs; (Hajj crowd).

c)Pedestrians holding hands to stay closer or assisting others across the roadway; (Group behavior in Hajj).

All the data concerning pedestrian walking speeds are illustrated in the paper [18] with other data taken from the literature surveillance which are entered in pedestrian database called PedGui, where the simulation input takes advantage from this results.

\subsection{Physical fitness (or level of exhaustion)}

The individual fitness or level of exhaustion can be affected by many internal factors like (sickness, disabilities, age, ...) and external factors like (weather, activities, surrounding conditions,...). For example fatigue is an important indication for the level of fitness for individuals and appear more clearly among older people than younger people. But the mechanisms describing this association in detail are not fully understood [19]. Decreasing habitual walking speed in older men and women is related to many factors that may be need to be examined. Many investigation from different science fields was conducted to quantify the walking speed and relaxation time of pedestrians of several ages under different conditions. The result of these studies indicates clearly that the individual walking speed is affected by the physiology and psychology of the individual.

In daily life, individual psychology will be affected by different kinds of stress. People become stressed when they feel that they are challenged or they need to cope with the current situation. Generally, stress is caused by a discrepancy between environmental demands and the abilities of individuals [20]. Stressors are what cause the stress, which can be a situation, an object or even another individual.

Investigations reveal that stress is made up of multiple things. Different people experience different aspects and identify with different definitions. The emotional or behavioral effect of stress is generally associated with increased aggression. This link can be found in both psychological models of emotion [21] and empirical studies of human behavior. However, the result of stress is not always negative. In some situations increased aggression can have positive effects, and can improve performance up to a specific point [22]. By measuring the connection between how people act (measured through recorded observations) and how people feel (measured indirectly via heart rate, skin temperature and self-reporting) psychologists have established a consequent relationship between increased stress and increased aggressive and agitated melancholia behavior [23]. This result has held across various stressors, different settings, cultures, and genders [24]. Our approach to simulate high density crowd dynamics was inspired by the results mentioned in this paragraph and take advantage of these empirical studies considering various stressors which affect the movement of pedestrians.

\subsection{Physical parameters}

The crowd behaviour is a very complex system driven by the individual decisions of agents with respect to their aims and destinations, environmental obstacles, and other surrounding circumstances. These physical parameters have a significant impact on the behaviour of the crowd in the case of an emergency. For accuracy and more realistic simulation, the physical factor must be considered in the input of the simulation. The size of the particle can be a determining factor in the reliability of the simulation results. 


\subsubsection{Pedestrian Radius Parameters}

This paragraph investigates some physical parameters and empirical data that was collected by Predtetschenski-Milinski [1]. Table 1 illustrates the projected area for different types of pedestrians.

Many measurements of persons with different ages and different clothes were made and the average values demonstrated in this table. The size of the individuals corresponded to the size of the individual's breast (thorax) height. For more realistic crowd simulation, the differences in human body size between all individuals or pedestrians in crowd must be considered and implemented with the correct percentage. For example, sport arenas or pop music concerts

concern mostly young fans and people. In the pilgrimage places, people are usually adults or

older people.

The individual's projected area on the ground is often referred as the pedestrian radius in crowd movement. This radius denoted by $R$ (called $r_{p}$ below in section 6 ) is a very important input in the simulation. The pedestrian radius depends on the weather factor. The radius of the individual in summer will be smaller as in winter, see table: 1 and fig: 1 . Some empirical data was collected by Predtetschenski-Milinski[1]. Table 1 illustrates the projected area of different types of pedestrians. Many measurements of people of different age and different clothes affected the average values demonstrated in this table.

The size of the individual corresponds to the size of the individual at breast (thorax) height.

Table 1. Set of different individual sizes [1].

\begin{tabular}{|l||l|}
\hline Type of Person & $\begin{array}{l}\text { Projected area } \\
\left(\mathrm{m}^{2}\right)\end{array}$ \\
\hline Children & $0.04-0.06$ \\
\hline Adolescent & $0.06-0.09$ \\
\hline $\begin{array}{l}\text { Grown-up in summer } \\
\text { clothes }\end{array}$ & 0.100 \\
\hline Grown-up in inter season & 0.113 \\
\hline Grown-up in winter clothes & 0.125 \\
\hline $\begin{array}{l}\text { Grown-up in inter season } \\
\text { clothes with briefcase }\end{array}$ & 0.180 \\
\hline $\begin{array}{l}\text { Grown-up in inter season } \\
\text { clothes with }\end{array}$ & 0.240 \\
\hline $\begin{array}{l}\text { Grown-up in inter season } \\
\text { clothes with }\end{array}$ & 0.360 \\
\hline
\end{tabular}

The parameters of a pedestrian flow and crowd density depend on the number of the persons composing the crowd. The measurements of the persons depends on their physical values, their age, and their clothes. Critical or dangerous situations will be demonstrated in the red cuboid, see fig:2, in this area the individual's body will be pressed or deformed.

\subsection{Climatic parameters (weather conditions)}

Man is not able to control the forces of nature, however, they can control and affect man. The forces of the nature are powerful and are able to influence people's behaviors. People prefer certain kinds of weather over others. For this reason and for the achievement of more realistic simulations, we must consider at least three different types of weather conditions that affect indirectly the pedestrian walking speed:

a)Dry: clear (no precipitation), with dry roads and dry pavements.

b) Rain: any type of rain from mist to moderate rain is not comfortable for walking people.

During heavy rain pedestrians tend to go faster inside.

c) Snow: when there is snow or ice in the atmosphere, on the road or pavement, or both, pedestrians take care and walk slowly to prevent slipping.

The weather parameter influences the velocity and the fitness (physical condition) of the individual. Richard L. Knoblauch [17] found relevant results about the functional relation between the pedestrian walking speed and weather condition. The data was collected on weekdays during daylight conditions. Surface conditions, weather conditions and the estimated wind intensity were documented for each observation. 
The analysis of the collected data showed that the mean walking speed was strongly affected by the weather and environmental factors. The collected data indicated also a relevant effect of age and of each the weather and environmental characteristics. This confirmed that weather plays a significant role in pedestrian movement in open air, hence the weather parameter must be considered in order to achieve more realistic simulations.

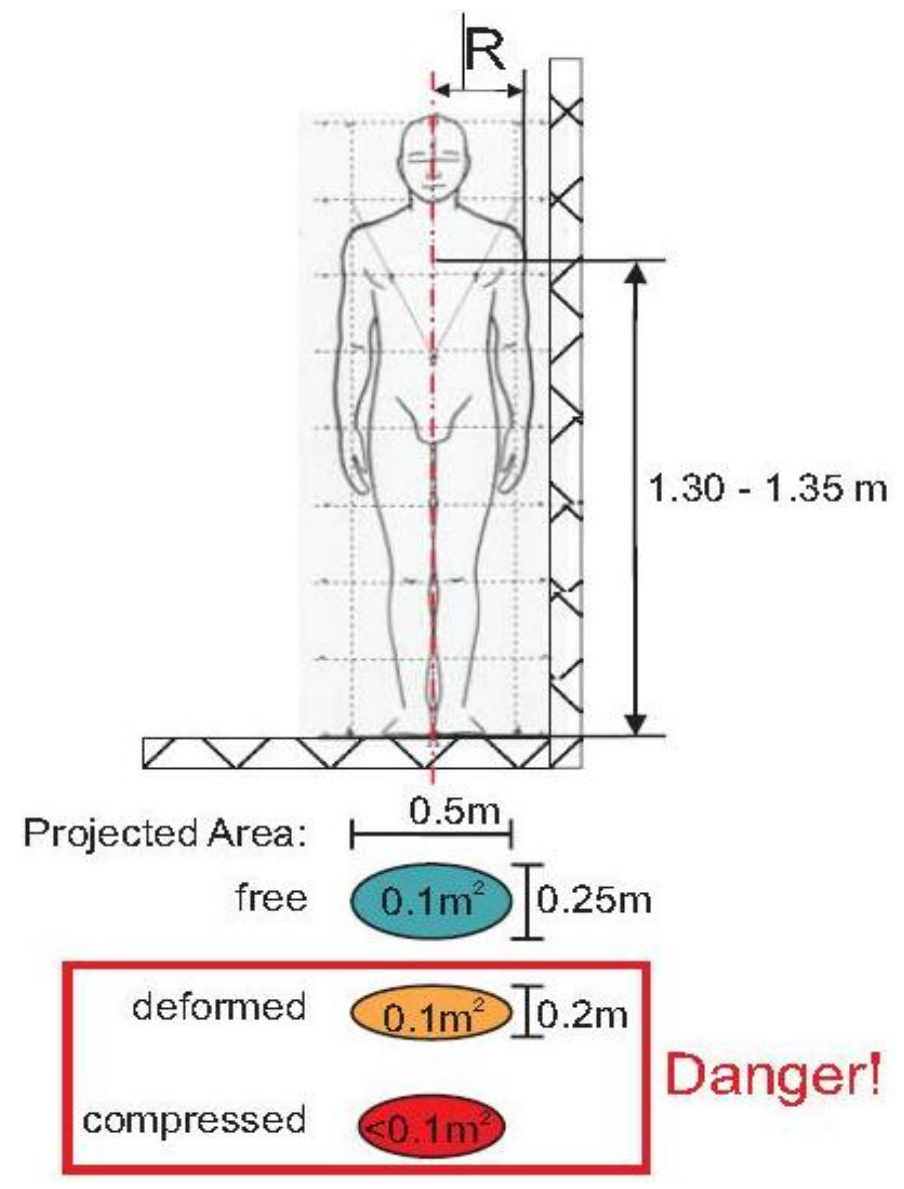

Figure 2: Projected area: Pedestrian Area Module (PAM), i.e. the surface A occupied by a pedestrian, is used as a more manageable unit $\left[\mathrm{m}^{2}\right]$. The PAM occupied by a motionless pedestrian can be calculated considering an elliptical or a rectangular form. According to Predtetschenski and Milinski [1], the average surface occupied by a motionless pedestrian is $A_{0}=w_{0} \cdot d_{0}$, where $\mathrm{w}_{0}$ and $\mathrm{d}_{0}$ are the average lateral width and depth of a human body taken in the height of the human shoulder (approximately $1.30 \mathrm{~m}$ above the ground) and measured not less than $0.6 \mathrm{~m}$. When a pedestrian is walking, a greater surface is required, that is, $A=w . d$. Both the terms $\mathrm{w}$ and $\mathrm{d}$ can be expressed as a function of the walking velocity $\mathrm{v}$. In addition, the lateral with could be made sensitive to the deck acceleration, since pedestrians tend to walk with their legs more widespread when the surface is laterally moving, as stressed by various authors (e.g. [25]).

\subsection{Psychology parameters}

Till this moment the psychological factor affecting the behavior of pedestrian are not completely understood. The decision making in human behavior at any time are affected by the psychology of the individual, however it is very important to consider the psychology factors in the simulation model [26]. Psychologists have extensively studied human characteristics and behaviors. Many investigations and researches show that differences in human behaviours are influenced by multiple factors, including differences in stimuli, physiological and cognitive state, social and cultural environment, previous life experiences and individual characteristics [27]. Despite this diversity, factors influencing direct human behaviors can be classified into some basic variations.

In the case of panic conditions or dangerous situations, the physiological state of individuals becomes very important in making decisions in a short time. Until now, it has been very difficult to describe and model the psychological state of pedestrians in normal and dangerous situations and to establish a quantitative relation between the walking speed and the mental state of individual. 


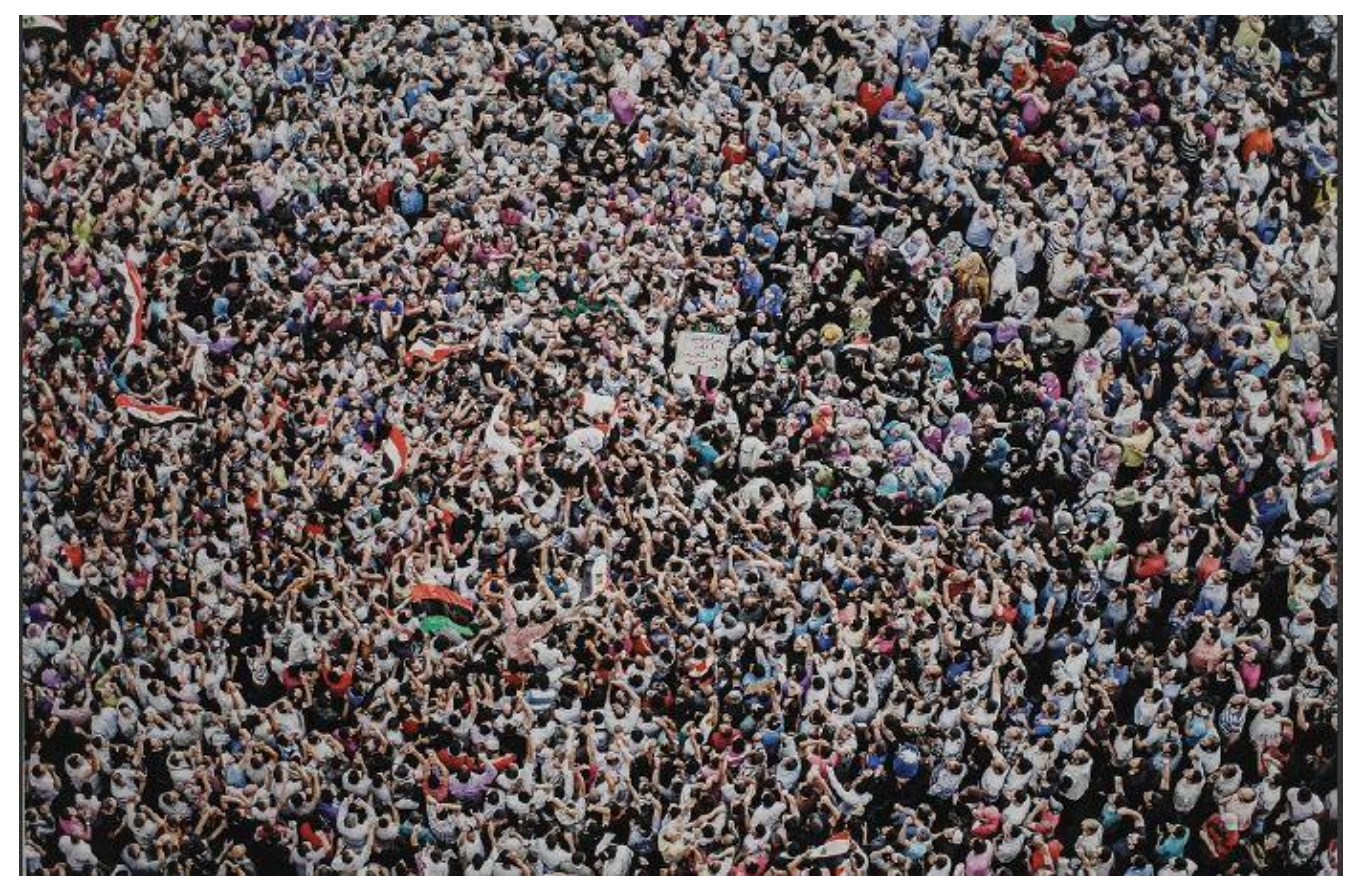

Figure 3. Crowd gathering to the Al-Tahrir place in Cairo (Arabic spring revolution). This crowd is referred as emotional crowd. A lot of physical and psychological parameters affecting the behaviour of the individuals within the crowd, (C) Kritisches-Netzwerk.

\section{Illustration of Parameters Effect on the Pedestrian Movement}

The motion of a single pedestrian at any time are governed by several parameters, the direction and speed will be the result of a long list of possible (and very likely conflicting) forces and circumstances. Since that, we mention the motivation to reach a certain place at a certain time (often referred to as will-force: time constraints, importance of punctuality, location constraints, importance of reaching a place and staying there long enough, etc.).

Changes in the desired velocity of a single pedestrian have been attributed to a number of factors, including body composition and functional capacity. Characteristic parameters that affected the movement of the fastest and slowest walkers are shown in schema 4 . The most important parameters and circumstances influencing the pedestrian's movement in emergent or normal behavior are demonstrated in the following graph.

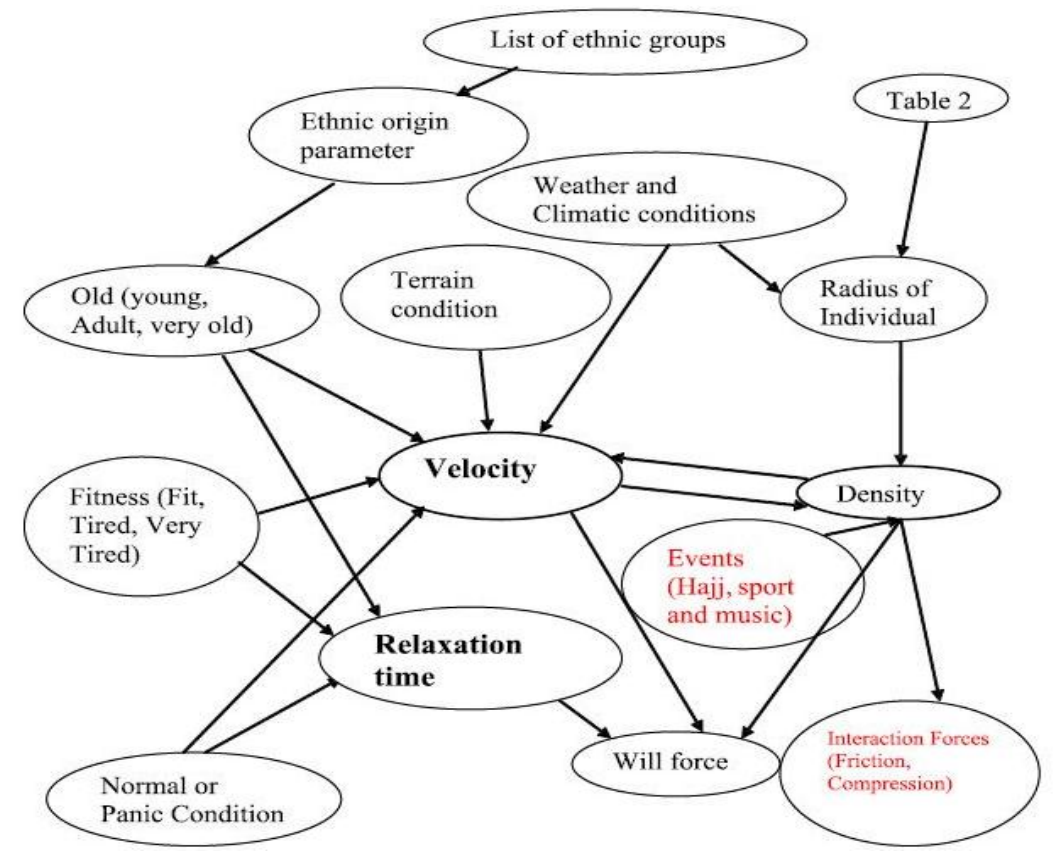

Figure 4. Graphical illustration of the list of circumstances influencing the pedestrian movements. 
In figure: 4 we demonstrate how will force $f_{w}$ and desired velocity $v_{d}$ as well relaxation time $\tau$ are influenced by parameters. It's clear that the movement of the pedestrians will be affected by the weather, fitness of the individual, geography,....

The average local speed is affected by the physical and mental state of the individuals. The different average projected body areas of people in different countries are very important to be considered, which has a significant impact on the average local density $\rho(r, t)$, average local speed $v(r, t)$ and the average local flow $Q(r, t)=\rho(r, t) v(r, t)$. In case of high pedestrian density, the friction and pressure forces can be affected by the mentioned effects above.

\section{Pedestrian Databases}

The evolution and design of pedestrian simulation models requires a lot of information and data.

All of these parameters (e.g. velocities and physical characteristics,...) are taken from observations of real pedestrian movement and their interactions at different events and places. The pedestrian parameters are taken from image data, or so called empirical data. This data is obtained using a video camera or human detectors. These parameters are derived from real-world observations.

They are very important in understanding the factors influencing how to alter crowd dynamics in different situations. The simulator input takes advantage of these parameters to achieve a certain realism in the simulation.

\subsection{Data Management}

The management of the huge amount of information and parameters concerning pedestrians requires the set-up of computer databases and test systems.

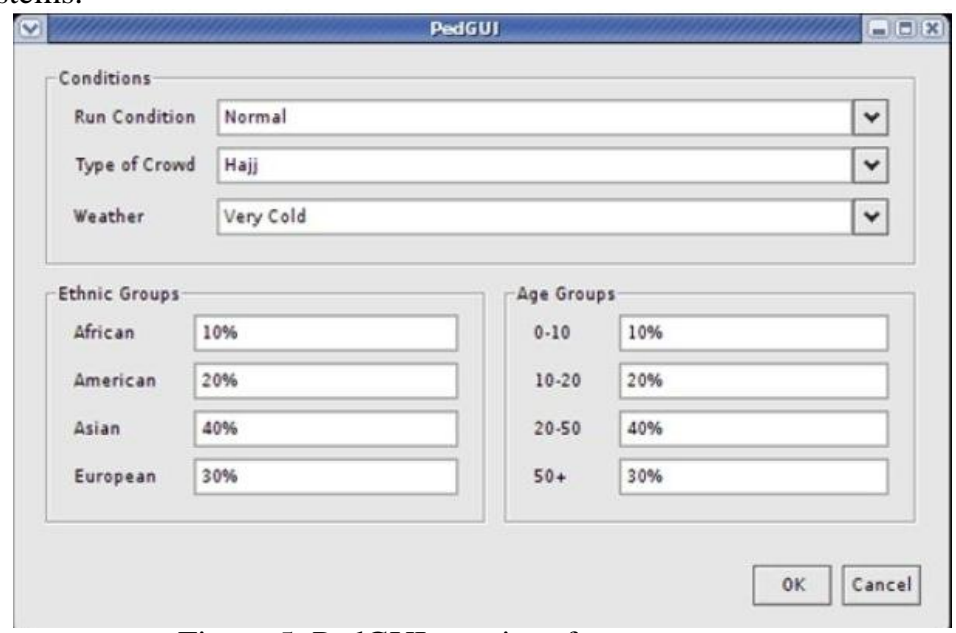

Figure 5. PedGUI user interface.

Fig: 5 shows the input of the simulation. These simulations represent complex interactions between individuals and their physical environment. For this reason considering all the parameters illustrated in the PedGUI can be advantageous for more realistic simulations. These factors attempt to predict pedestrian movement in both normal and panic situations, and in the PedGUI these options will be considered. The percentage of men and women, old and young people, and the ethnic origin of the pedestrians can be considered in the crowd simulation input. With the help of this system we can achieve a great deal, firstly more realism in the simulation and secondly a database of different ethnic groups that can be used for other purposes and studies.

\section{Simulation Using One Type of Pedestrian}

Our first set of simulations consisted a pedestrian flow through the Mataf area (see fig. 6 (A)). The Mataf area was 105 $\mathrm{m}$ wide and $154 \mathrm{~m}$ long and in the center of the Mataf the Kaaba with $21.14 \mathrm{~m}$ long and $11.53 \mathrm{~m}$ wide (see fig. 6 (B)) is placed. The circumambulation area (ground floor) of Mataf is around 16170 square meters. In this set of simulation one type of pedestrian is considered, that's mean at the beginning all pedestrian have the same desired velocity, the same radius and the same relaxation time. Each pedestrian's desired speed was set to a relatively high value of $1.2 \mathrm{~m} / \mathrm{s}$, the pedestrian radius $r_{p}$ was $0.178 \mathrm{~m}$ to $0.2 \mathrm{~m}$ and the relaxation time $\tau$ was set in the range of $0.5 \mathrm{~s}$ to $1 \mathrm{~s}$. The pedestrian streaming the Mataf floor through three different ways denoted by 1, 2, 3 in the figure 6 (B). After a pedestrian finishes the Tawaf (seven times circling around the Kaaba) they are going in the direction of Sa'y by the exit or gate 4. The most seen effect in the simulation snapshot is the congested area declaring the beginning and the end of the Tawaf movement, this reflects a part of the reality but not all the reality, the vacant places in the Mataf floor or disturbance fluctuation in the pedestrian flow, especially near the Kaaba wall, have not to do with the reality, thus the video taking reveals 
something else. This can be traced back to the interaction forces between the particles, the fact that all particles have the same repulsive forces and block each other creates afterwards a vacant places.

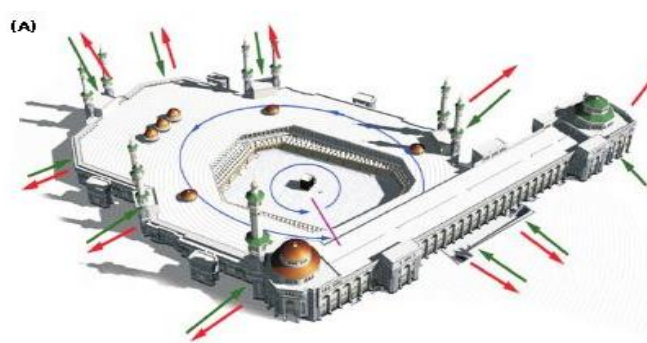

(B)

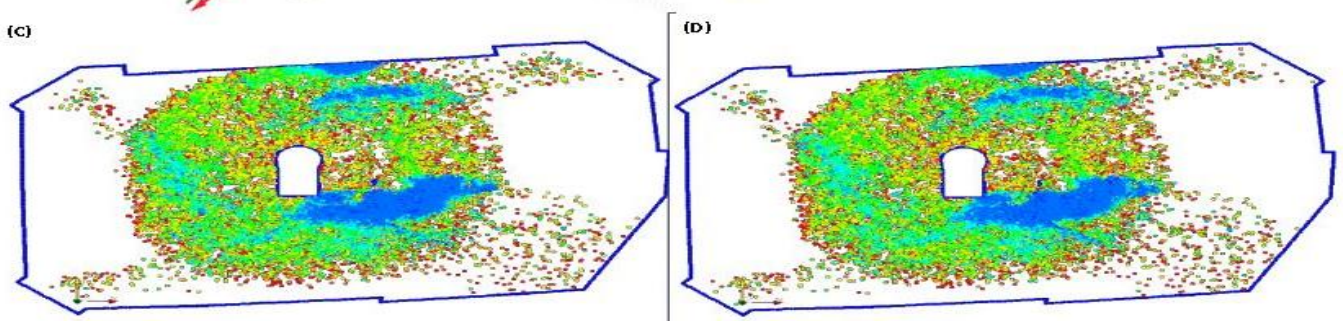

Figure 6: (A) 3D model displaying the geometry form of the Haram mosque building; (B) CAD drawing displaying the ground Mataf floor, 1,2,3 indicates the pedestrian influx and 4 indicate the pedestrian outflow; (C) and (D)

two consecutive snapshot PedFlow.

\section{Simulation Using PedGui}

For more realistic simulation results many effects must be considered in the simulation input. The physical parameters describing the individual fitness or level of exhaustion are found in a table in the appendix. The table presents different categories of pedestrians with different culture origins and different fitness levels. The fitness levels of individuals can be determined through the relaxation time parameter $\tau$. This parameter reflects the fitness level and the age of the individuals in the crowd, considering the fact that elderly people need more time to reach their desired velocity than young people. The other relevant parameter is the particle size or so-called pedestrian radius $r_{p}$, which has a significant impact on the average local density $\rho(r, t)$ and consequently the average local pedestrian flow in this area. A set of different pedestrian radii is presented in table 1 . This parameters can be associated with weather condition parameters. Obviously people in warm weather climates tend to dress very differently than those originating from a region with winter temperatures. Consequently the Pedestrian Area Module (PAM), i.e. the occupation area by a single individual, see fig. 2, in winter is evidently bigger than in the summer. Our initial attempts to establish a real crowd consists of a set of agents with different physical properties, i.e. mean agents with different initial parameters as shown in the table in the appendix. This manoeuvre helps to reduce the disturbances caused by the interaction forces between the particles and to reproduce a more realistic pedestrian flow. Different types of pedestrians with different social, cultural and ethical background are considered in the simulation input. Either we try to introduce the individual fitness or level of exhaustion.

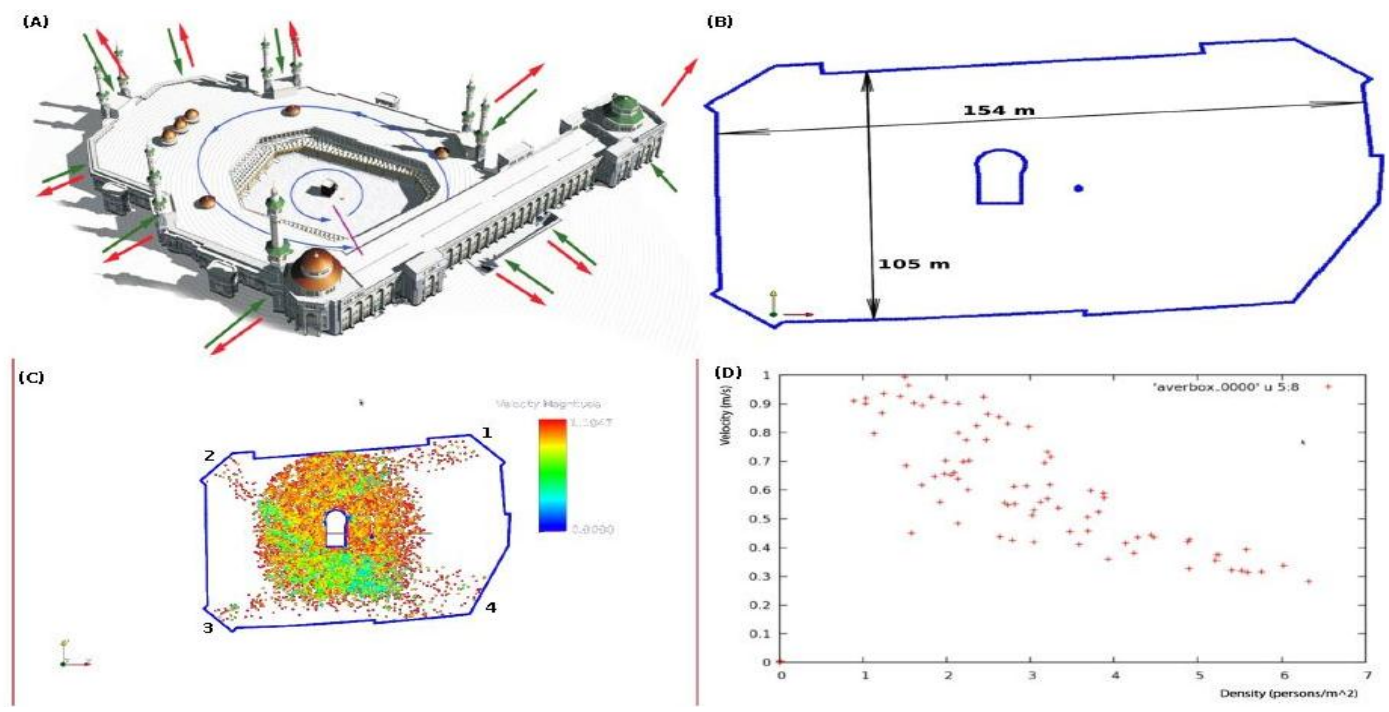

Figure 7: (A) 3D model displaying the geometry form of the Haram mosque building; (B) CAD drawing displaying the 
ground Mataf floor, 1,2,3 indicates the pedestrian influx and 4 indicate the pedestrian outflow; (C) and (D)

two consecutive snapshot PedFlow.

For the first moment one can not realise the difference in the results illustrated in figure 6 and figure 7. Both pictures exhibit the same critical points. Note that the pedestrian density is very high at the places where the Tawaf begins and ends. Note the clumping of pedestrians going in opposing directions, when the pilgrims finish the Tawaf. One may conclude that the different characters at the influx have no influence on the movement of pilgrims, but this is not true and this phenomenon is demonstrated in figure 6. One can clearly see vacant places in the Mataf area, and this results from a repulsive interaction between the particles. This effect is better seen in the case of high crowd density. Due to the higher pressure in case of high density crowd the repulsive forces between the individual become very considerable opposite forces that the resultant is the complete stop of the movement, than appears this vacant region in the walking floor.

\section{Conclusion}

This paper presented a number of physical and psychological parameters that affect the movement of individuals in a crowd. Moreover it is an attempt to collect parameters based on observed collective behaviour in emergency conditions, where the detailed design of interactions is overlapped by group behaviour. We present many attributes describing a more complex behaviour of pedestrian in both indoor and outdoor environments. These parameters can be very important to improve simulation tools and achieve more realistic results, its mean produce results that can be compared favourably with the real data.

In the microscopic framework, people are treated as individual entities (particles). The individuals are modelled as particles interacting through attractive and repulsive forces. The evolution of the particles in time is determined by physical and social laws and influenced by a lot of attributes which describe the interaction among the particles as well as their interactions with the physical surroundings.

As presented in fig.4 force is an essential aspect of crowd models and affected by a myriad of external attributes e.g the fitness level and age of individual affect the relaxation time $\tau$, in fact young people have better reflection as old people. Bio-physical experiments have been undertaken to investigate the human body under extreme physical stress conditions. The radius of particle or called physical parameter play significant roll in the crowd density. Moreover we try to understand how the human body will be deformed or compressed in cases of critical crowd densities.

A database contain a lot of data concerning the pedestrian characteristics was established. All collected empirical data from different way, automatic and manual methods was stored in a table called PedGui. We have analysed video recordings of the crowd movement in the Tawaf within the mosque in Mecca during the Hajj of the 27th November, 2009 [18]. We have evaluated unique video recordings of a $105 \times 154 \mathrm{~m}$ large Mataf area taken from the roof of the Mosque, where up to 3 million Muslims perform the Tawaf and Sa'y rituals within 24 hours. The collected data about the mean walking speed for the younger pedestrians ranged from 1.37 to $1.57 \mathrm{~m} / \mathrm{s}$ for very low pedestrian density, with an overall mean speed of $1.47 \mathrm{~m} / \mathrm{s}$ either the means for the older pedestrians range from $1.13 \mathrm{~m} / \mathrm{s}$ to $1.26 \mathrm{~m} / \mathrm{s}$, with an overall mean speed of $1.192 \mathrm{~m} / \mathrm{s}$ was incorporated in the PedGui database. The microscopic walking speed for a pedestrian moving in the pilgrim stream around the Kabba was $1.0816 \mathrm{~m} / \mathrm{s}$ at the edge of the Mataf where the pedestrian density was below 3 persons $/ \mathrm{m}^{2}$ and it was $0.2676 \mathrm{~m} / \mathrm{s}$ for a density around 5 to 6 persons $/ \mathrm{m}^{2}$ inside the Mataf. This result was compatible with the statistical results of Predtetschenski and Milinski [1]. For the simulation results the average speed of the system decreases as the percentage of the pedestrian increases in the area. Comparing the data resulting from the simulation with empirical observations data one can assert the generated data that are consistent with empirical data across a wide range of characteristics.

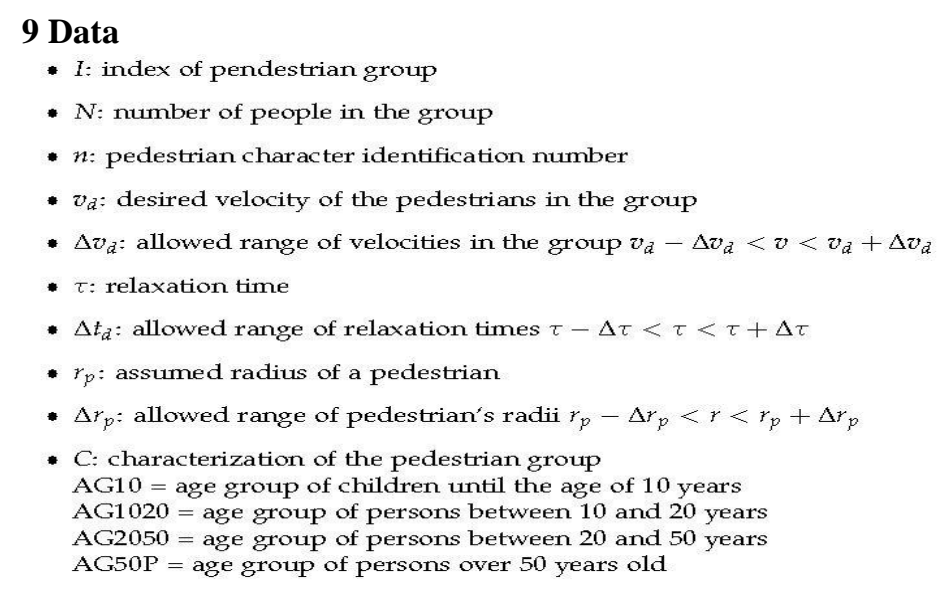




\begin{tabular}{|c|c|c|c|c|c|c|c|c|c|}
\hline$I$ & $N$ & $n$ & $v_{d}$ & $\Delta v_{d}$ & $\tau$ & $\Delta \tau$ & $r_{p}$ & $\Delta r_{p}$ & C \\
\hline 1 & 0 & 1 & 1.46 & 0.200 & 0.4 & 0.000 & 0.099792 & 0.050 & African Fit AG10 \\
\hline 2 & 30 & 1 & 1.46 & 0.200 & 0.4 & 0.000 & 0.099792 & 0.050 & African Fit AG10 \\
\hline 3 & 0 & 1 & 1.4572 & 0.200 & 0.5 & 0.000 & 0.1219 & 0.050 & African Fit AG1020 \\
\hline 4 & 30 & 1 & 1.4572 & 0.200 & 0.5 & 0.000 & 0.1219 & 0.050 & African Fit AG1020 \\
\hline 5 & 0 & 1 & 1.4572 & 0.200 & 0.5 & 0.000 & 0.1409 & 0.050 & African Fit AG2050 \\
\hline 6 & 30 & 1 & 1.4572 & 0.200 & 0.5 & 0.000 & 0.1409 & 0.050 & African Fit AG2050 \\
\hline 7 & 0 & 1 & 1.2095 & 0.200 & 0.7 & 0.000 & 0.1409 & 0.050 & African Fit AG50P \\
\hline 8 & 30 & 1 & 1.2095 & 0.200 & 0.7 & 0.000 & 0.1409 & 0.050 & African Fit AG50P \\
\hline 9 & 0 & 1 & 1.3115 & 0.200 & 0.3600 & 0.000 & 0.0997 & 0.050 & African Tired AG10 \\
\hline 10 & 30 & 1 & 1.3115 & 0.200 & 0.3600 & 0.000 & 0.0997 & 0.050 & African Tired AG10 \\
\hline 11 & 0 & 1 & 1.3115 & 0.200 & 0.45 & 0.000 & 0.1219 & 0.050 & African Tired AG1020 \\
\hline 12 & 30 & 1 & 1.3115 & 0.200 & 0.45 & 0.000 & 0.1219 & 0.050 & African Tired AG1020 \\
\hline 13 & 0 & 1 & 1.3115 & 0.200 & 0.45 & 0.000 & 0.1409 & 0.050 & African Tired AG2050 \\
\hline 14 & 30 & 1 & 1.3115 & 0.200 & 0.45 & 0.000 & 0.1409 & 0.050 & African Tired AG2050 \\
\hline 15 & 0 & 1 & 1.0885 & 0.200 & 0.63 & 0.000 & 0.1409 & 0.050 & African Tired AG50P \\
\hline 16 & 30 & 1 & 1.0885 & 0.200 & 0.63 & 0.000 & 0.1409 & 0.050 & African Tired AG50P \\
\hline 17 & 0 & 1 & 1.1658 & 0.200 & 0.3200 & 0.000 & 0.0997 & 0.050 & African Very Tired AG10 \\
\hline 18 & 30 & 1 & 1.1658 & 0.200 & 0.3200 & 0.000 & 0.0997 & 0.050 & African Very Tired AG10 \\
\hline 19 & 0 & 1 & 1.1658 & 0.200 & 0.4 & 0.000 & 0.1219 & 0.050 & African Very Tired AG1020 \\
\hline 20 & 30 & 1 & 1.1658 & 0.200 & 0.4 & 0.000 & 0.1219 & 0.050 & African Very Tired AG1020 \\
\hline 21 & 0 & 1 & 1.1658 & 0.200 & 0.4 & 0.000 & 0.1409 & 0.050 & African Very Tired AG 2050 \\
\hline 22 & 30 & 1 & 1.1658 & 0.200 & 0.4 & 0.000 & 0.1409 & 0.050 & African Very Tired AG 2050 \\
\hline 23 & 0 & 1 & 0.9676 & 0.200 & 0.5599 & 0.000 & 0.1409 & 0.050 & African Very Tired AG50P \\
\hline 24 & 30 & 1 & 0.9676 & 0.200 & 0.5599 & 0.000 & 0.1409 & 0.050 & African Very Tired AG50P \\
\hline 25 & 0 & 1 & 1.3247 & 0.200 & 0.4 & 0.000 & 0.1219 & 0.050 & American Fit AG10 \\
\hline 26 & 30 & 1 & 1.3247 & 0.200 & 0.4 & 0.000 & 0.1219 & 0.050 & American Fit AG10 \\
\hline 27 & 0 & 1 & 1.3247 & 0.200 & 0.5 & 0.000 & 0.1490 & 0.050 & American Fit AG 1020 \\
\hline 28 & 30 & 1 & 1.3247 & 0.200 & 0.5 & 0.000 & 0.1490 & 0.050 & American Fit AG1020 \\
\hline 29 & 0 & 1 & 1.3247 & 0.200 & 0.5 & 0.000 & 0.1723 & 0.050 & American Fit AG2050 \\
\hline 30 & 30 & 1 & 1.3247 & 0.200 & 0.5 & 0.000 & 0.1723 & 0.050 & American Fit AG2050 \\
\hline 31 & 0 & 1 & 1.0995 & 0.200 & 0.7 & 0.000 & 0.1723 & 0.050 & American Fit AG50P \\
\hline 32 & 30 & 1 & 1.0995 & 0.200 & 0.7 & 0.000 & 0.1723 & 0.050 & American Fit AG50P \\
\hline 33 & 0 & 1 & 1.1923 & 0.200 & 0.3600 & 0.000 & 0.1219 & 0.050 & American Tired AG10 \\
\hline 34 & 30 & 1 & 1.1923 & 0.200 & 0.3600 & 0.000 & 0.1219 & 0.050 & American Tired AG10 \\
\hline 35 & 0 & 1 & 1.1923 & 0.200 & 0.45 & 0.000 & 0.1490 & 0.050 & American Tired AG1020 \\
\hline 36 & 30 & 1 & 1.1923 & 0.200 & 0.45 & 0.000 & 0.1490 & 0.050 & American Tired AG1020 \\
\hline 37 & 0 & 1 & 1.1923 & 0.200 & 0.45 & 0.000 & 0.1723 & 0.050 & American Tired AG 2050 \\
\hline 38 & 30 & 1 & 1.1923 & 0.200 & 0.45 & 0.000 & 0.1723 & 0.050 & American Tired AG 2050 \\
\hline 39 & 0 & 1 & 0.9896 & 0.200 & 0.63 & 0.000 & 0.1723 & 0.050 & American Tired AG50P \\
\hline 40 & 30 & 1 & 0.9896 & 0.200 & 0.63 & 0.000 & 0.1723 & 0.050 & American Tired AG50P \\
\hline 41 & 0 & 1 & 1.0598 & 0.200 & 0.3200 & 0.000 & 0.1219 & 0.050 & American Very Tired AG10 \\
\hline 42 & 30 & 1 & 1.0598 & 0.200 & 0.3200 & 0.000 & 0.1219 & 0.050 & American Very Tired AG10 \\
\hline 43 & 0 & 1 & 1.0598 & 0.200 & 0.4 & 0.000 & 0.1490 & 0.050 & American Very Tired AG1020 \\
\hline 44 & 30 & 1 & 1.0598 & 0.200 & 0.4 & 0.000 & 0.1490 & 0.050 & American Very Tired AG1020 \\
\hline
\end{tabular}




\begin{tabular}{|c|c|c|c|c|c|c|c|c|c|}
\hline 45 & 0 & 1 & 1.0598 & 0.200 & 0.4 & 0.000 & 0.1723 & 0.050 & American Very Tired AG2050 \\
\hline 46 & 30 & 1 & 1.0598 & 0.200 & 0.4 & 0.000 & 0.1723 & 0.050 & American Very Tired AG2050 \\
\hline 47 & 0 & 1 & 0.8796 & 0.200 & 0.5599 & 0.000 & 0.1723 & 0.050 & American Very Tired AG50P \\
\hline 48 & 30 & 1 & 0.8796 & 0.200 & 0.5599 & 0.000 & 0.1723 & 0.050 & American Very Tired AG50P \\
\hline 49 & 0 & 1 & 1.4572 & 0.200 & 0.4 & 0.000 & 0.099792 & 0.050 & Asian Fit AG10 \\
\hline 50 & 30 & 1 & 1.4572 & 0.200 & 0.4 & 0.000 & 0.0997 & 0.050 & Asian Fit AG10 \\
\hline 51 & 0 & 1 & 1.4572 & 0.200 & 0.5 & 0.000 & 0.1219 & 0.050 & Asian Fit AG1020 \\
\hline 52 & 30 & 1 & 1.4572 & 0.200 & 0.5 & 0.000 & 0.1219 & 0.050 & Asian Fit AG1020 \\
\hline 53 & 0 & 1 & 1.4572 & 0.200 & 0.5 & 0.000 & 0.1409 & 0.050 & Asian Fit AG2050 \\
\hline 54 & 30 & 1 & 1.4572 & 0.200 & 0.5 & 0.000 & 0.1409 & 0.050 & Asian Fit AG2050 \\
\hline 55 & 0 & 1 & 1.2095 & 0.200 & 0.7 & 0.000 & 0.1409 & 0.050 & Asian Fit AG50P \\
\hline 56 & 30 & 1 & 1.2095 & 0.200 & 0.7 & 0.000 & 0.1409 & 0.050 & Asian Fit AG50P \\
\hline 57 & 0 & 1 & 1.3115 & 0.200 & 0.3600 & 0.000 & 0.0997 & 0.050 & Asian Tired AG10 \\
\hline 58 & 30 & 1 & 1.3115 & 0.200 & 0.3600 & 0.000 & 0.0997 & 0.050 & Asian Tired AG10 \\
\hline 59 & 0 & 1 & 1.3115 & 0.200 & 0.45 & 0.000 & 0.1219 & 0.050 & Asian Tired AG1020 \\
\hline 60 & 30 & 1 & 1.3115 & 0.200 & 0.45 & 0.000 & 0.1219 & 0.050 & Asian Tired AG1020 \\
\hline 61 & 0 & 1 & 1.3115 & 0.200 & 0.45 & 0.000 & 0.1409 & 0.050 & Asian Tired AG2050 \\
\hline 62 & 30 & 1 & 1.3115 & 0.200 & 0.45 & 0.000 & 0.1409 & 0.050 & Asian Tired AG2050 \\
\hline 63 & 0 & 1 & 1.0885 & 0.200 & 0.63 & 0.000 & 0.1409 & 0.050 & Asian Tired AG50P \\
\hline 64 & 30 & 1 & 1.0885 & 0.200 & 0.63 & 0.000 & 0.1409 & 0.050 & Asian Tired AG50P \\
\hline 65 & 0 & 1 & 1.1658 & 0.200 & 0.3200 & 0.000 & 0.0997 & 0.050 & Asian Very Tired AG10 \\
\hline 66 & 30 & 1 & 1.1658 & 0.200 & 0.3200 & 0.000 & 0.0997 & 0.050 & Asian Very Tired AG10 \\
\hline 67 & 0 & 1 & 1.1658 & 0.200 & 0.4 & 0.000 & 0.1219 & 0.050 & Asian Very Tired AG1020 \\
\hline 68 & 30 & 1 & 1.1658 & 0.200 & 0.4 & 0.000 & 0.1219 & 0.050 & Asian Very Tired AG1020 \\
\hline 69 & 0 & 1 & 1.1658 & 0.200 & 0.4 & 0.000 & 0.1409 & 0.050 & Asian Very Tired AG2050 \\
\hline 70 & 30 & 1 & 1.1658 & 0.200 & 0.4 & 0.000 & 0.1409 & 0.050 & Asian Very Tired AG2050 \\
\hline 71 & 0 & 1 & 0.9676 & 0.200 & 0.5599 & 0.000 & 0.1409 & 0.050 & Asian Very Tired AG50P \\
\hline 72 & 30 & 1 & 0.9676 & 0.200 & 0.5599 & 0.000 & 0.1409 & 0.050 & Asian Very Tired AG50P \\
\hline 73 & 0 & 1 & 1.3247 & 0.200 & 0.4 & 0.000 & 0.11088 & 0.050 & European Fit AG10 \\
\hline 74 & 30 & 1 & 1.3247 & 0.200 & 0.4 & 0.000 & 0.11088 & 0.050 & European Fit AG10 \\
\hline 75 & 0 & 1 & 1.3247 & 0.200 & 0.5 & 0.000 & 0.13552 & 0.050 & European Fit AG1020 \\
\hline 76 & 30 & 1 & 1.3247 & 0.200 & 0.5 & 0.000 & 0.13552 & 0.050 & European Fit AG1020 \\
\hline 77 & 0 & 1 & 1.32479 & 0.200 & 0.5 & 0.000 & 0.15664 & 0.050 & European Fit AG2050 \\
\hline 78 & 30 & 1 & 1.3247 & 0.200 & 0.5 & 0.000 & 0.15664 & 0.050 & European Fit AG2050 \\
\hline 79 & 0 & 1 & 1.0995 & 0.200 & 0.7 & 0.000 & 0.15664 & 0.050 & European Fit AG50P \\
\hline 80 & 30 & 1 & 1.0995 & 0.200 & 0.7 & 0.000 & 0.15664 & 0.050 & European Fit AG50P \\
\hline 81 & 0 & 1 & 1.1923 & 0.200 & 0.3600 & 0.000 & 0.11088 & 0.050 & European Tired AG10 \\
\hline 82 & 30 & 1 & 1.1923 & 0.200 & 0.3600 & 0.000 & 0.11088 & 0.050 & European Tired AG10 \\
\hline 83 & 0 & 1 & 1.1923 & 0.200 & 0.45 & 0.000 & 0.13552 & 0.050 & European Tired AG1020 \\
\hline 84 & 30 & 1 & 1.1923 & 0.200 & 0.45 & 0.000 & 0.13552 & 0.050 & European Tired AG1020 \\
\hline 85 & 0 & 1 & 1.1923 & 0.200 & 0.45 & 0.000 & 0.15664 & 0.050 & European Tired AG2050 \\
\hline 86 & 30 & 1 & 1.1923 & 0.200 & 0.45 & 0.000 & 0.15664 & 0.050 & European Tired AG2050 \\
\hline 87 & 0 & 1 & 0.9896 & 0.200 & 0.63 & 0.000 & 0.15664 & 0.050 & European Tired AG50P \\
\hline 88 & 30 & 1 & 0.9896 & 0.200 & 0.63 & 0.000 & 0.15664 & 0.050 & European Tired AG50P \\
\hline 89 & 0 & 1 & 1.0598 & 0.200 & 0.3200 & 0.000 & 0.11088 & 0.050 & European Very Tired AG10 \\
\hline 90 & 30 & 1 & 1.0598 & 0.200 & 0.3200 & 0.000 & 0.11088 & 0.050 & European Very Tired AG10 \\
\hline 91 & 0 & 1 & 1.0598 & 0.200 & 0.4 & 0.000 & 0.13552 & 0.050 & European Very Tired AG1020 \\
\hline 92 & 30 & 1 & 1.0598 & 0.200 & 0.4 & 0.000 & 0.13552 & 0.050 & European Very Tired AG1020 \\
\hline 93 & 0 & 1 & 1.0598 & 0.200 & 0.4 & 0.000 & 0.15664 & 0.050 & European Very Tired AG2050 \\
\hline 94 & 30 & 1 & 1.0598 & 0.200 & 0.4 & 0.000 & 0.15664 & 0.050 & European Very Tired AG2050 \\
\hline 95 & 0 & 1 & 0.8796 & 0.200 & 0.5599 & 0.000 & 0.15664 & 0.050 & European Very Tired AG50P \\
\hline 96 & 30 & 1 & 0.8796 & 0.200 & 0.5599 & 0.000 & 0.15664 & 0.050 & European Very Tired AG50P \\
\hline
\end{tabular}

\section{References}

Anderson, C. A. (2001) Heat and violence. Current Directions in Psychological Science, 10(1), 33-38.

Berkowitz, L. (1990). On the formation and regulation of anger and aggression: A Cognitiveneo Associationistic analysis. American Psychologist, 45(4), 494-503, 
Chattaraj, U., Seyfried, A., \& P. Chakroborty. Comparison of pedestrian fundamental diagram across cultures. Advances in complex systems, 12, 393-405, 2009.

Coleman, J. S. (1994). Foundations of Social Theory. Belknap Series. Belknap Press of Harvard University Press; http://books.google.de/books?id=a4D18tiX4b8 C, 1994.

Dallard, P., Fitzpatrick, T. Flint, Le Bourva, A. S. Low, A. Ridsdill, R. M., \& Willford, M.(2001). The London Millennium Footbridge. The Structural Engineer, 79(22), 17-33.

Elliott, D., \& Smith, D. (1993). Football stadia disasters in the United Kingdom: Learning from tragedy? Industrial and Environmental Crisis Quarterly, 7(3), 205-229, 1993.

Evans, G. W. (1984). Environmental Stress. CUP Archive.

Eysenck, M. Simply psychology. Psychology Press.

Fruin, J. J. (1987).Pedestrian planning and design. Elevator World A. Seyfried. Comparison of pedestrian fundamental diagram across cultures. Advances in Complex Systems, 12(3), 393-405, 2009.

Helbing, D. I. Farkas, J. Molnar, P., \& Vicsek, T. Simulation of pedestrian crowds in normal and evacuation situations. In Pedestrian and evacuation dynamics, 21, 21-58.Springer, Berlin, 2002.

Helbing, D. Johansson, A., \& Zein Al-Abideen, H. (2007).The dynamics of crowd disasters: An empirical study. Phys. Rev. E, 75.

Jacobs, B. D., \& Hart, P. (1992). Disaster at Hillsborough Stadium: A comparative analysis. In:Parker, D. J. \& Handmer, J. W. (eds.) Hazard management and emergency planning, Chapt 10. James and James Science, London.

Keating, J. P. (1982).The myth of panic. Fire Journal, 147, 57-61.

Kessler, H. Crowd panic: Proactive versus reactive responses. Technical report, International Foundation for Protection Officers,1996-2013.http://www.ifpo.org/resources/articlesand-reports/protection-of-specific-environments/ crowd-panic-proactive-versus-reactiveresponses/.

Knoblauch, R. L. Pietrucha, M. T., \& Nitzburg, M. (1996) Field studies of pedestrian walking speed and start-up time. Transportation Research Record 1538. Washington (DC): National Research Council, Transportation Research Board, 7-38.

M. Mänty, C. F., de Leon, T., Rantanen, P., Era, A. Pedersen, N. Ekmann, A., Schroll, M., \& Avlund, K.(1978). Mobility-related fatigue, walking speed, and muscle strength in older people. Oxford Journals: Medicine \& Health \& Science \& Mathematics: The Journals of Gerontology: SeriesA, 67A, 5, 523-529, 2011. T. Cox. Stress. London: Macmillan Press.

Mintz, A. (1951). Non-adaptive group behavior. Journal of Abnormal Psychology, 46, 150-159.

Mohamed H. Dridi. (2015)Tracking individual targets in high density crowd scenes analysis of a video recording in hajj 2009. Current Urban Studies (CUS), 3(1).

Pelechano, K. O’brien, Silverman, B., \& Badler, N.(2005). Simulation incorporating agent psychological models, roles and communication. In 1st Int'l Workshop on Crowd Simulation, 21-30.

Predtechenski, W. M., \& Milinski, A. I. (1971)Personenströme in Gebäuden. Verlagsgesellschaft Rudolf Müller, Köln.

Quarantelli, E. L. (1957). The Behavior of Panic Participants. National Emergency Training Center: http://books.google.de/books?id=tSAJSQAACAA J.

Rutherford, F. J., \& Ahlgren, A. (1991). Science for All Americans. Oxford University Press, USA; http://books.google.de/books?id=LKadiKAUljEC

Smith, R. A., \& Dickie, J. F. (1993). Engineering for crowd safety: proceedings of the International Conference on Engineering for Crowd Safety, London, UK, 17-18 March. Elsevier;http://books.google.de/books?id=Rs VRAAAAMAAJ, 1993.

Turner, R. H., \& Killian, L. (1987). M. Collective behavior. Prentice Hall College Div, 3rd edition.

Yerkes, R. M., \& Dodson, J. D. (1990). The relation of strength of stimulus to rapidity of habitformation. Journal of Comparative Neurology and Psychology, 18(5), 459-482.

\section{(c) $\mathbf{E Y}$}

This work is licensed under a Creative Commons Attribution 3.0 License. 